in vivo $35: 1901-1905$ (2021)

doi:10.21873/invivo.12455

\title{
Endoventricular Pericardial Patch Repair of a Giant Left Posterior Ventricular Aneurysm
}

\author{
OVIDIU STIRU ${ }^{1,2}$, ROXANA CARMEN GEANA ${ }^{1}$, REZA NAYYERANI $^{1}$, ANDREEA SIMINA CHIBULCUTEAN ${ }^{1}$, \\ ADRIAN TULIN ${ }^{3}$, LAURA RADUCU ${ }^{4,5}$, NICOLAE BACALBASA ${ }^{6,7}$, IRINA BALESCU ${ }^{8}$, CAMELIA DIACONU $^{9,10}$, \\ FLORENTINA FURTUNESCU ${ }^{11}$, DANIEL RADAVOI ${ }^{12,13}$ and VLAD ANTON ILIESCU ${ }^{1,2}$ \\ ${ }^{1}$ Emergency Institute for Cardiovascular Diseases “Prof. Dr. C.C. Iliescu”, Bucharest, Romania; \\ ${ }^{2}$ Department of Cardio-Thoracic Pathology, "Carol Davila" \\ University of Medicine and Pharmacy, Bucharest, Romania; \\ ${ }^{3}$ Department of Anatomy, "Carol Davila" University of Medicine and Pharmacy, Bucharest, Romania; \\ ${ }^{4}$ Department of Plastic and Reconstructive Microsurgery, "Carol Davila" \\ University of Medicine and Pharmacy, Bucharest, Romania; \\ ${ }^{5}$ Department of Plastic and Reconstructive Surgery, "Prof Dr. Agripa Ionescu" \\ Clinical Emergency Hospital, Bucharest, Romania; \\ ${ }^{6}$ Department of Visceral Surgery, Center of Excellence in Translational \\ Medicine "Fundeni" Clinical Institute, Bucharest, Romania; \\ ${ }^{7}$ Department of Obstetrics and Gynecology, "Carol Davila" \\ University of Medicine and Pharmacy, Bucharest, Romania; \\ ${ }^{8}$ Department of Surgery "Ponderas" Academic Hospital, Bucharest, Romania; \\ ${ }^{9}$ Department of Internal Medicine, "Carol Davila” University of Medicine and Pharmacy, Bucharest, Romania; \\ ${ }^{10}$ Department of Internal Medicine, Clinical Emergency Hospital of Bucharest, Bucharest, Romania; \\ ${ }^{11}$ Department of Public Health and Management University of Medicine \\ and Pharmacy "Carol Davila”, Bucharest, Romania; \\ ${ }^{12}$ Department of Urology, "Prof. Dr. Th. Burghele” Clinical Hospital, Bucharest, Romania; \\ ${ }^{13}$ Department of Urology, "Carol Davila" University of Medicine and Pharmacy, Bucharest, Romania
}

\begin{abstract}
Background/Aim: Left ventricular aneurysms are complications following acute myocardial infarction. Left posterior left ventricular aneurysms occurring in a submitral position constitute a minor entity, and those leaving the mitral apparatus intact are extremely rare. Case Report: Herein, we report the case of a 58-year-old patient with a past medical history of coronary artery disease and myocardial infarction with a giant left posterior left ventricular aneurysm with moderate mitral valve incompetence. Results: The patient underwent myocardial
\end{abstract}

This article is freely accessible online.

Correspondence to: Nicolae Bacalbasa, "Carol Davila" University of Medicine and Pharmacy, 37 Dionisie Lupu Street, 020021 Bucharest, Romania. Tel: +40 723540426, e-mail: nicolae_bacalbasa@yahoo.ro

Key Words: Giant left ventricular aneurysm, submitral Aneurysm, pericardial bovine patch, myocardial revascularization. revascularization and, through a transaneurysmal approach, successful endoventricular pericardial patch wall reconstruction with no impact on mitral valve competence. Conclusion: Whenever the mitral valve is not affected, a trans aneurysmal approach with endoventricular pericardial patch in association with myocardial revascularization represents a safe and reproducible approach with good functional outcomes.

In the last years, the incidence of the postinfarction left ventricular aneurysms (LVA) is decreasing, and the prognosis of coronary artery disease (CAD) and myocardial infarction (MI) is improved by the increased use of thrombolysis or acute percutaneous coronary intervention (PCI) (1). The apex and the posterior wall are the most common sites of aneurysm formation; as few as $1 \%$ of ventricular aneurysms affect the ventricle's lateral wall (2). However, postinfarction LVA remains a serious disorder with a varied clinical presentation depending on size, location, and valvular involvement. LVA can cause many complications, including congestive heart failure, ventricular arrhythmia, systemic embolism, and 


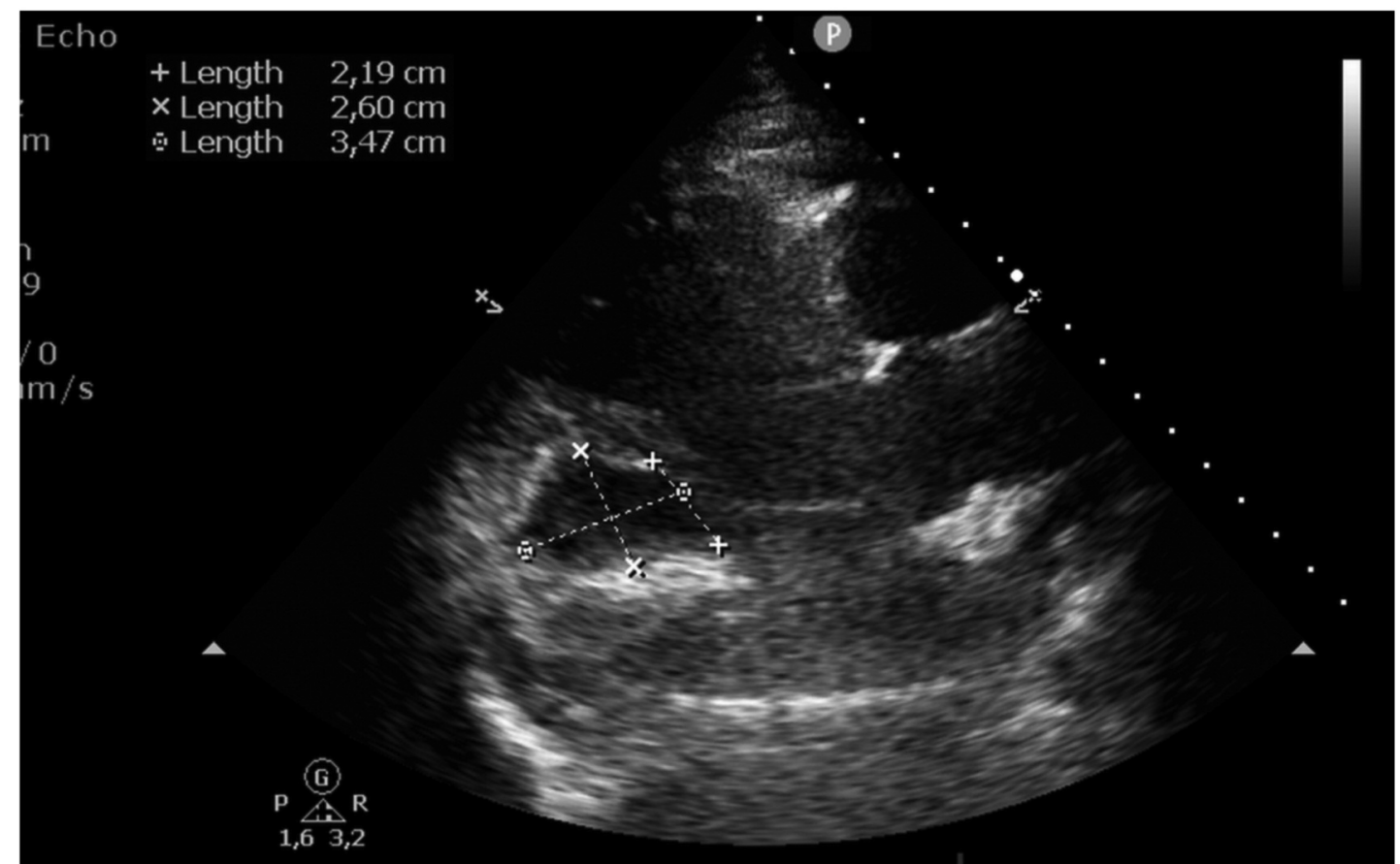

Figure 1. Transesophageal intraoperative echocardiogram, to assess the left posterior left ventricular aneurysm (LPVA) before surgical repair (34/26 mm).

ventricular rupture. Left posterior left ventricular aneurysms (LPVA) profoundly alter cardiac geometry, distort the mitral ring, and cause mitral valve incompetence. Surgical treatment is indicated in cases of LVA complicated with angina pectoris, malignant ventricular arrhythmia, recurrent embolization from the left ventricle (LV), or mitral valve regurgitation. The goal of surgical intervention is to resect the aneurysm, correct the size and geometry of the LV, restore valvular mitral competence, coronary artery bypass graft (CABG), and improve left ventricular function. Aneurysm resection with endoventricular pericardial patch repair and concurrent $\mathrm{CABG}$ is effective and reasonable of the different possible surgical approaches (1-3).

\section{Case Report}

A 58-year-old female was admitted to our clinic with progressive chest pain radiating to the left arm, fatigue, dizziness, and dyspnea on exertion, worsening for the last period. This patient had relevant cardiovascular risk factors (systemic hypertension, obesity, dyslipidemia, smoker, age). She was treated for heart failure at another hospital. Her medical history included left ventricular pseudoaneurysm, ischemic heart disease, poster-inferolateral myocardial infarction 18 months previously, moderate mitral valve regurgitation, heart failure New York Heart Association (NYHA) II, pulmonary obstructive chronic disease, liver hemangioma, hiatal hernia, and vitiligo. On admission, the patient was hemodynamically stable. Her systemic blood pressure was 120/60 $\mathrm{mmHg}$ (pulse rate $65 \mathrm{bpm}$ ), oxygen saturation levels $97-98 \%$ in atmospheric air, the respiratory rate was 20 breaths per minute, and the body mass index was 39.8. The electrocardiogram (ECG) showed sinus rhythm. Laboratory evaluation was notable for glucose $175 \mathrm{mg} / \mathrm{dl}$ triglycerides $234 \mathrm{mg} / \mathrm{dl}$, High Density Lipoprotein-cholesterol (HDL-cholesterol) $40 \mathrm{mg} / \mathrm{dl}$, Low Density Lipoproteincholesterol (LDL-cholesterol) $133 \mathrm{mg} / \mathrm{dl}$, uric acid $9.1 \mathrm{mg} / \mathrm{dl}$. Transthoracic echocardiography (TTE) revealed a 35/26mm posterolateral aneurysm of the left ventricle (Figure 1) and a left ventricular ejection fraction (FE) of $40 \%$. Color-flow Doppler TEE showed minimal mitral regurgitation (MR). Coronary angiography showed severe coronary disease with an $80 \%$ stenosis of the first segment on the left anterior descending coronary artery (LAD) and total occlusion of segment two of the right coronary artery. Contrast-enhanced cardiac magnetic resonance imaging (MRI) and the results were as follows: large aneurysmal dilatation on the inferior and lateral-inferior wall of the left ventricle, with large inlet, measuring $60 \mathrm{~mm}$ deep and $60 \mathrm{~mm}$ (across) in diameter with a significant layer of thrombus (Figure 2). It was placed very close to the mitral annulus. On MRI exam, the left ventricle was dilated with moderate systolic dysfunction. The right ventricle had normal dimensions and function. Early capture of Gadolinium evidenced voluminous thrombus in the 


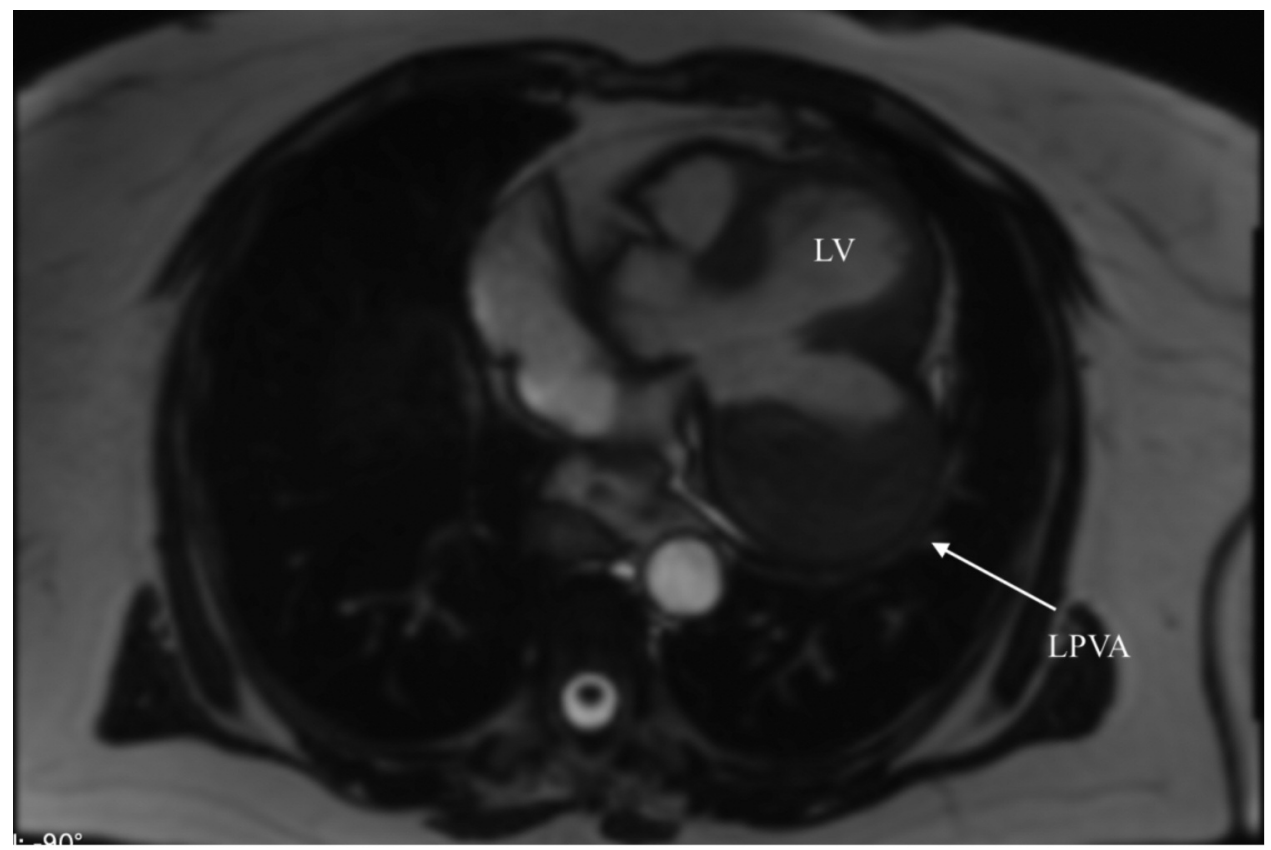

Figure 2. Magnetic resonance imaging (MRI) showing the left posterior ventricular aneurysm measuring 60/60 mm LPVA.

pseudoaneurysm, and the late capture exposed interruptions of myocardial continuity, suggesting an LV aneurysm. Although the aneurysmal inlet was very close to the mitral valve, the mitral annulus was not dilated, and no significant mitral valve insufficiency was seen. Also, the MRI revealed transmural myocardial infarction in the territory of the right coronary artery without residual myocardial viability. The surgical intervention was proposed to the patient, and she accepted. Open repair of the aneurysm through a midline sternotomy was planned (Figure 3). Standard cardiopulmonary bypass (CPB) with aortobicaval cannulation and anterograde cardioplegic arrest allowed performing surgery. Longitudinal incision was performed parallel to the posterior descending artery of the LPVA, and cavity thrombus was carefully removed. Visual inspection and palpation identified the border between the scar tissue and the contractile myocardium. The mitral subvalvular apparatus was found intact, mitral annulus was found not dilated, and the native valve was considered preservable. Thinned left aneurysmal ventricular wall was resected, leaving a border area $<2 \mathrm{~cm}$ from the scar tissue to enable the reconstruction of normal left ventricular geometry, the ventricular opening remains greater than $3 \mathrm{~cm}$ diameter. The closure is achieved with the Dor technique-endoventricular circular patch (Figure $4 \mathrm{a}$ and $\mathrm{b}$ ). The bovine pericardial patch size was not amenable to a specific formula or calculation and was determined only by the ventricular opening's size. The remaining left aneurysmal wall was closed with Teflon felt

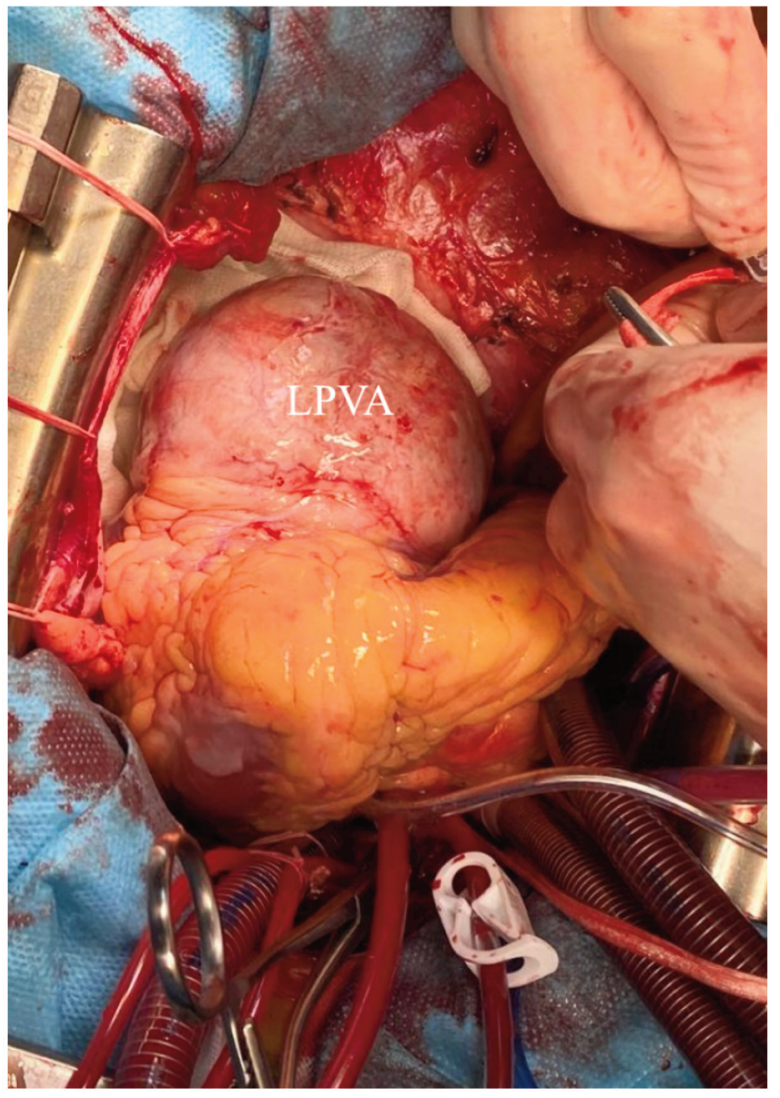

Figure 3. Intraoperative view of the unruptured left posterior left ventricular aneurysm (LPVA). 


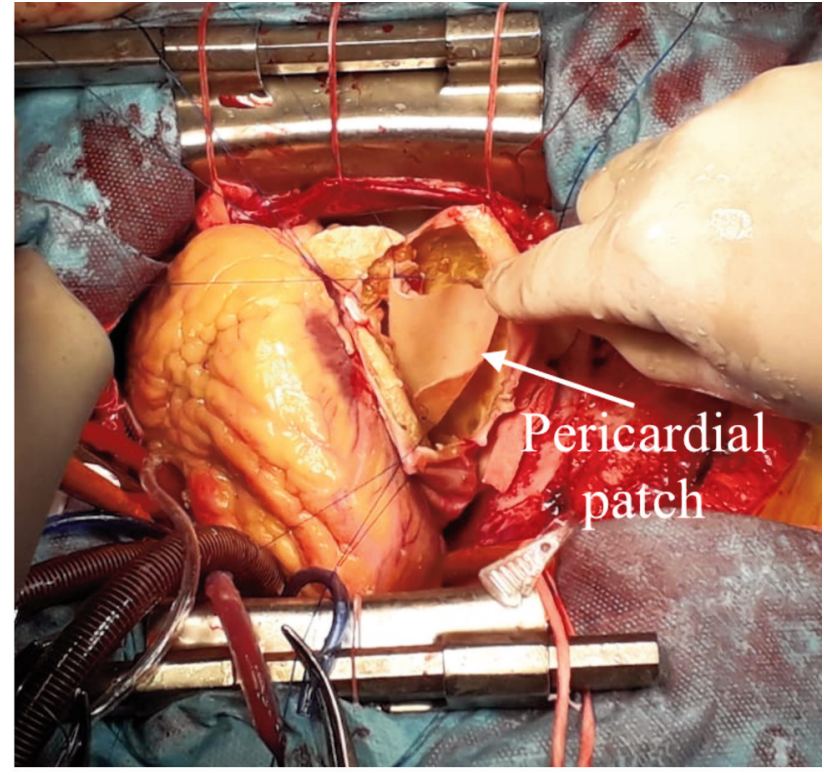

Figure 4. Intraoperative view - opened left posterior left ventricular aneurysm (LPVA).

strips (Figures 5 and 6). Myocardial revascularization was performed using the left internal mammary artery (LIMA) to bypass the LAD II. The time of CPB was $68 \mathrm{~min}$ and aortic clamp time was $43 \mathrm{~min}$. Uneventful weaning from CPB. Transesophageal echocardiogram (TEE) showed that the ventricular ejection fraction remains $45 \%$, and mitral insufficiency was mild. Her postoperative course was uneventful, the intensive care unit stay was three days, and she was discharged to her home on the ten postoperative days. Further follow-up at six months on TTE confirmed preserved left ventricular function and durability of the repair.

\section{Discussion}

Most LVA have been reported after acute transmural myocardial infarctions (AMI). Untreated LVA can have a mortality of up to $50 \%$. (3). There has been more frequency in anterior than in inferior locations. In our case, transmural myocardial infarction due to occlusion of the dominant right coronary artery led to the LPVA formation. LVA begins to develop in a few days after AMI, with an incidence of 30-35\% after one year (4-7). Intracavitary thrombus affects approximately $40-60 \%$ of patients (8-10). Thromboembolic event is considerable after the third month in patients with ventricular septal aneurysms (VSA) (8). Small LVA is usually asymptomatic and is typically diagnosed by routine TTE. When the aneurysms increase in size, patients develop phenomena of heart failure, angina, and manifestations such as acute pulmonary edema, thromboembolism, and ventricular

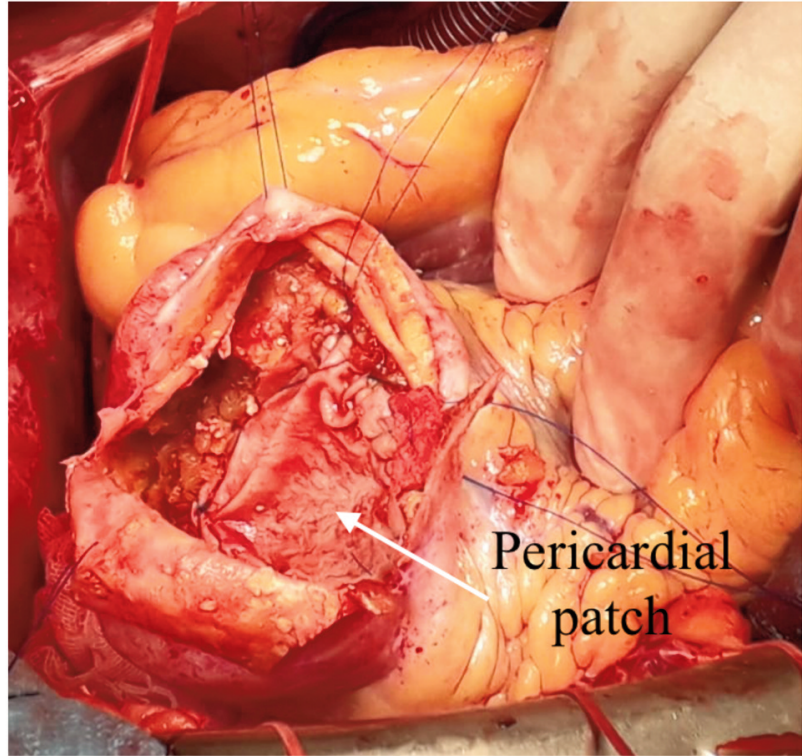

Figure 5. Intraoperative view of surgical reconstruction of aneurysmal neck with bovine pericardial patch.

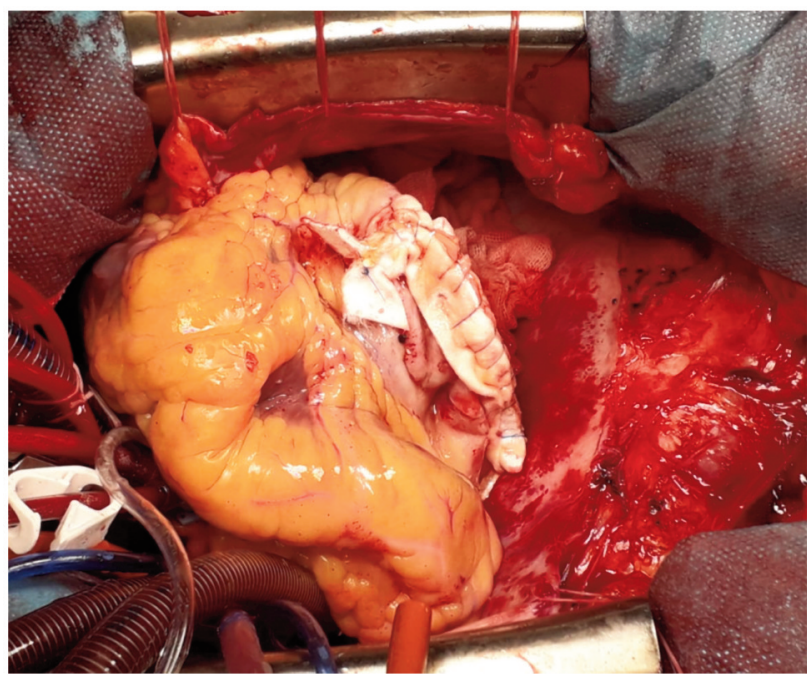

Figure 6. Final aspect - the remaining left aneurysmal wall was closed with Teflon felt strips.

rupture $(6,7)$. The patient described in this case report had a documented myocardial infarction 18 months ago. Since the infarction, her condition has progressively deteriorated, with the predominance of heart failure and angina pectoris. However, rarer than anterior aneurysms, posterior or posterobasal aneurysms occur in approximately $11 \%$ of LVA and can present a surgical challenge (3). Preoperative planning leads 
to successful surgical outcomes, routine TTE reveals LPVA, and color-flow echocardiographic imaging identifies abnormal flow within the aneurysm, the presence of layered thrombus, and involvement of the mitral valve. MRI provides excellent LPVA measurements and provides indications of the viability of myocardial tissue and extent and effect on left ventricular and mitral valve geometry. Coronarography and ventriculography provide additional information on coronary heart disease (11). Surgical procedures are the preferred treatment for all locations of LVA and hold perioperative mortality up to $10 \%$ (9). Technique such as plication, excision/suture, and ventricular patch reconstruction is indicated and must be adapted individual for each patient (5, 10). LPVA endoventricular patch plasty has been described using a left ventriculotomy and through a transatrial approach. The transatrial approach (this is not our case) was first described for patients with a small-neck posterior aneurysm, predominantly in African patients without ischemic heart disease (12). Left ventricular reconstruction was first described by Vincent Dor in 1984 using endoventricular patch reconstruction still has excellent results today for LPVA with ischemic etiology (3). The surgery key is patch reconstruction without affecting the mitral valve ring and preserves size and geometry of the left ventricular. However, we consider that there are two limitations and difficulties which should be taken in consideration when performing such a surgical procedure. The first one is related to the left ventricle's preserved function and revascularization of the existing coronary lesions while the second one is dictated by the need or not for the correction of mitral regurgitation.

\section{Conclusion}

Thorough diagnosis, preoperative evaluation, and appropriate surgical technique lead to successful surgical outcomes. In selected cases when the mitral valve was not affected, the trans aneurysmal approach with endoventricular pericardial patch concomitant with myocardial revascularization allows for a simple, safe and reproducible approach.

\section{Conflicts of Interest}

The Authors have no conflicts of interest to declare regarding this study.

\section{Authors' Contributions}

OS, VAI, RCG, ASC performed the surgical procedure; NB, IB, AT, $\mathrm{DR}$ reviewed literature data; $\mathrm{RN}, \mathrm{CD}$, ASC preoperative investigation of the patient; FF,DR postoperative follow up of the patient; RN, ASC, RCG, LR prepared the draft of the manuscript; VAI was advisor of the surgical procedures; OS, NB, VAI reviewed the final version of the manuscript. The Authors read and approved the final version of the manuscript.

\section{References}

1 Lundblad R, Abdelnoor M and Svennevig JL: Surgery for left ventricular aneurysm: Early and late survival after simple linear repair and endoventricular patch plasty. J Thorac Cardiovasc Surg 128(3): 449-456, 2004. PMID: 15354107. DOI: 10.1016/j.jtcvs.2004.04.017

2 Habertheuer A, Andreas M, Wiedemann D, Rath C and Kocher A: Giant lateral left ventricular wall aneurysm sparing the submitral apparatus. J Cardiothorac Surg 8: 201, 2013. PMID: 24172071. DOI: $10.1186 / 1749-8090-8-201$

3 Dor V, Di Donato M, Sabatier M, Montiglio F, Civaia F and RESTORE Group: Left ventricular reconstruction by endoventricular circular patch plasty repair: A 17-year experience. Semin Thorac Cardiovasc Surg 13(4): 435-447, 2001. PMID: 11807739. DOI: 10.1053/stcs.2001.29966

4 Gueron M, Wanderman KL, Hirsch $M$ and Borman J: Pseudoaneurysm of the left ventricle after myocardial infarction: A curable form of myocardial rupture. J Thorac Cardiovasc Surg 69(5): 736-742, 1975. PMID: 1127971.

5 Ruzza A, Czer LSC, Arabia F, Vespignani R, Esmailian F, Cheng W, De Robertis MA and Trento A: Left ventricular reconstruction for postinfarction left ventricular aneurysm: Review of surgical techniques. Tex Heart Inst J 44(5): 326-335, 2017. PMID: 29259502. DOI: 10.14503/THIJ-16-6068

6 Achenbach S, Ropers D and Daniel WG: Calcified left ventricular aneurysm. N Engl J Med 348(24): 2469, 2003. PMID: 12802039. DOI: 10.1056/NEJM200306123482419

7 Evangelou D, Letsas KP, Gavrielatos G, Alexanian IP, Pappas LK, Sioras E and Kardaras F: Giant left-ventricular pseudoaneurysm following silent myocardial infarction. Cardiology 105(3): 137-138, 2006. PMID: 16428885. DOI: 10.1159/000091071

8 Strecker T, Baum U, Harig F, Niedobitek G, Mahmoud FO and Weyand M: Visualization of a large ventricular aneurysm in a young man by 16 -slice multi-detector row spiral computed tomography before successful surgical treatment. Int $\mathrm{J}$ Cardiovasc Imaging 22(3-4): 537-541, 2006. PMID: 16273312. DOI: $10.1007 / \mathrm{s} 10554-005-9041-\mathrm{z}$

9 Sattar Y and Alraies MC: Ventricular aneurysm. [Updated 2020 Nov 26]. In: StatPearls [Internet]. Treasure Island (FL): StatPearls Publishing; 2021.

10 Loures D, Carvalho R, Lima jr. J, Costa M, Tiszka A, Dergint A, AbrÃo M, Stahlke P, Wolker R, Schmidlin C and BrommelstrÖet $\mathrm{M}$ : Tratamento cirúrgico dos aneurismas de ventrículo esquerdo e isquemia coronária. Revista Brasileira de Cirurgia Cardiovascular 12(2): 122-131, 2011. DOI: 10.1590/ S0102-76381997000200004

11 Dorobantu LF, Stiru O, Prodea A, Cioranu R, Georgescu A, Filipescu D and Iliescu VA: Unique case of primary malignant fibrous histiocytoma of the right ventricle with moderator band involvement. Heart Surg Forum 14(4): E245-E248, 2011. PMID: 21859644. DOI: 10.1532 /HSF98.20101154

12 Antunes MJ: Submitral left ventricular aneurysms. Correction by a new transatrial approach. J Thorac Cardiovasc Surg 94(2): 241-245, 1987. PMID: 3613623.

Received March 7, 2021

Revised March 27, 2021

Accepted April 1, 2021 